23

\section{EPIDEMIOLOGY OF BLOODSTREAM INFECTIONS IN THE FIRST YEAR AFTER PEDIATRIC LUNG TRANSPLANTATION.}

LA Danziger-Isakov, The Children's Hospital, The Cleveland Clinic Foundation, Cleveland, OH; Washington University, St. Louis, MO.

Background: Significant morbidity and mortality associated with infection occurs in the first year after pediatric lung transplantation. To better understand the clinical significance of bloodstream infections (BSI), we systematically evaluated the epidemiology of BSI in the first year posttransplant. Methods: A retrospective case-cohort study of pediatric primary lung transplant recipients was performed. The incidence of BSI and organisms isolated were determined through medical and laboratory record review. We assessed variation in causative organisms and rate of BSI in three time periods after transplantation: acute ( $0-30$ days), intermediate (31-90 days) and late ( $91-365$ days) Results: From July 1990 to November 2000, 190 pediatric patients received primary lung transplants. Twenty-six percent (49 of 190) of recipients had at least one BSI. The most common organisms isolated were Coagulase-negative staphylococcus $(\mathrm{n}=25,28.4 \%)$. Pseudomonas aeruginosa $(\mathrm{n}=14$, $16.0 \%$ ), and Candida species ( $\mathrm{n}=9,10.2 \%)$. The overall rate of BSI was 2.1 per 1000 catheter-days. The highest rate of BSI occurred in the acute period compared to the intermediate and late periods a $5.5,1.3$ and 1.6 per 1000 catheter-days, respectively $(P=0.21)$. Early BSI was associated with death in the first year after transplant $(\mathrm{RR}=3.9,95 \% \mathrm{CI} 1.6-9.4, \mathrm{p}=0.002)$. Conclusions: BSI occur frequently after primary pediatric lung transplantation with the highest rate in the first 30 days afte transplantation. Early BSI is associated with death in the first year after transplant. Prevention of early BSI could potentially decrease mortality after pediatric lung transplantation. Mendeloff, Medical City Hospital, Dallas, TX; S Sweet, M delaMorena, C Huddleston, MR DeBaun,

26

THE EFFECTS OF A PRACTICE CHANGE IN THE UMBILICAL ARTERIAL CATHETER REGIMEN FOR LESS THAN 800-GRAM INFANTS.

JK Jackson, TL Sandritter, J Raucci, HW Kilbride; Children's Mercy Hospital and Clinics, UMKC School of Medicine, Kansas City, MO

Background: We have previously demonstrated through a randomized study that in comparison to a hyptonic regimen, an isotonic amino acid (IAA) umbilical arterial catheter (UAC) solution/flush regimen is associated with less hemolysis, improved nutrition and alterations in fluid/electrolyte balance. We hypothesized that the difference in fluid/electrolyte balance was contributed to by differences in renal function in the 2 groups in relation to the generation of plasma free hemoglobin secondary to hemolysis. Since implementation of findings from a controlled study may be met with unpredictable obstacles and results, we have continued to monitor data as the isotonic regimen was introduced into practice. Objective: To assess the introduction of an IAA UAC infusion/flush regimen into general practice and to evaluate fluid/electrolyte status in infants before (group 1) and after (group 2) implementation in an effort to delineate underlying factors related to fluid electrolyte balance in infants $<800 \mathrm{~g}$ BW. Design/Methods: This was a retrospective review of information collected for QI purposes. Patients, $<800 \mathrm{~g}$ with UAC in place, were identified from 2 available databases. IRB approval was obtained The data was analyzed to determine differences in serum sodium $(\mathrm{Na})$, potassium $(\mathrm{K})$, blood urea nitrogen, (BUN), creatinine (Cr); fluid balance, BUN:Cr ratio; and line complications. Based on data from our previous study, 24 infants in each group would provide powers of $99 \%, 94 \%$, and $94 \%$ ( $\dot{a}=0.05$ ) for sodium levels, intakes, and urine outputs, respectively. Results: The data of 33 and 24 infants were evaluated from the time period before and after the change in practice respectively. The new practice was implemented in $86 \%$ of cases. There were no group differences in demographics. Mean GA (range) 25 wks (22-28), BW $651 \mathrm{~g}(435-782)$, days with UAC $5.7(1-13)$. Na level was higher and $\mathrm{K}$ level lower in group 2 in the $1^{\text {st }} 2$ days of life. There was no difference in UAC complications, $\mathrm{Cr}$, BUN levels, urinary output or volume intake. BUN:Cr ratio was higher in group 2 in comparison to group 1 ( $25 \pm 7$ vs $21 \pm 9$, respectively; $P<.05)$. There was also a significant difference in the number of critical high and low Na values between the two groups. Conclusion: The difference in BUN:Cr ratio supports the theory of improved renal function resulting in relative volume depletion in the IAA UAC group. Other group differences were not as pronounces as in the controlled monitoring introduction of practices from controlled trials.

\section{4}

RENAL FUNCTION IN RARE LIVING RELATED SMALL BOWEL TRANSPLANT CHILDREN.

E John, A Lumpaopong, S Kecskes, G Arteaga, G Testa, E Benedetti, Department of Pediatrics and Surgery, University of Illinois at Chicago, Chicago, Illinois

BACKGROUND: Data from United Network for Organ Sharing (UNOS) and Organ Procurement and Transplantation Network (OPTN) reveals that living related small bowel transplant (LRSMT) in children, age less than 5 years, has been performed only 5 patients since $1988-2004$. From these national data, 4/5 LRSMT were done at The University of Illinois Medical Center. In this study, we present renal function in this group of patients. METHODS: Retrospective review of renal function was done in 3 patients: 4 LRSMT (1 patient underwent retransplant). Mean aged at LRSMT was $2.31 \pm 1.5$ years $(1-4$ years). Mean follow up period was $11.5 \pm 8.3$ months ( $4-21$ months). All patients had underlying gastroschisis and short bowel syndrome. Immunosuppressive regimen included tacrolimus and steroid therapy. Serum electrolyte, glomerular and tubular functions were measured at 1,3,6,9,12 and 18 months after transplant. Creatinine clearance $(\mathrm{CrCl})$ was estimated by Schwartz formula and tubular functions were estimated by calculating fractional excretion of sodium (FENa, normal $<1 \%$ ), magnesium (FEMg, normal $2-2.5 \%$ ), urine calcium/creatinine ratio $(\mathrm{UCa} / \mathrm{Cr}$, normal $<0.21$ ) and tubular reabsorption of phosphorus (TRP, normal $80-90 \%$ ). Proteinuria was diagnosed when urine protein/creatinine (Uprot/Cr) $>0.2$. RESULTS: Patient survival was $100 \%$. One patient lost graft secondary to PTLD at 4 months post LRSMT and underwent combined liver-small bowel transplant 8 months later. $\mathrm{CrCl}$ at $1,3,6,9,12$ and 18 months was $92.2 \pm 16$, $98.1 \pm 25,95.2 \pm 5,108.5 \pm 2,112.5(\mathrm{n}=1)$, and $113.9(\mathrm{n}=1) \mathrm{cc} / \mathrm{min} / 1.73 \mathrm{~m}^{2}$ respectively. Proteinuria and tubular dysfunctions were observed after LRSMT and gradually improve. (Uprot/ $\mathrm{Cr}=1.49 \pm 1.0$, $\mathrm{FENa}=2.3 \pm 0.9 \%, \mathrm{UCa} / \mathrm{Cr}=0.20 \pm 0.2, \mathrm{FEMg}=15.7 \pm 7.8 \%$, and $\mathrm{TRP}=76.6 \pm 19.3 \%$ at month and $\mathrm{Uprot} / \mathrm{Cr}=1.27 \pm 0.7, \mathrm{FENa}=1.1 \pm 1.0 \%, \mathrm{UCa} / \mathrm{Cr}=0.11 \pm 0.1, \mathrm{FEMg}=13.8 \pm 11.4 \%$, and TRP $=87.0 \pm 17.1 \%$ at 3 months). All patients had renal tubular acidosis, hypocalcemia, hypomagnesemia and hypophosphatemia and received bicarbonate, calcium, magnesium and phospypomagnesemia and hypophosphatemia and received bicarbonate, calcium, magnesium and phosphorus supplement. Tacrolimus level at $1,3,6,9,12$ and 18 months was $17.0 \pm 2,9.6 \pm 3,11.7 \pm$
$1,12.8 \pm 1,12.5(\mathrm{n}=1) \mathrm{ng} / \mathrm{ml}$ respectively. CONCLUSION: Abnormal glomerular, tubular function and electrolyte imbalance was observed after LRSMT especially in the early phase. Serial monitoring of renal function and long term follow up is necessary in this patient group.

\section{5}

\section{BARRIERS TO SCREENING INFANTS FOR RETINOPATHY OF PREMATU- RITY AFTER DISCHARGE OR TRANSFER FROM A NEONATAL INTEN- SIVE CARE UNIT.}

M Attar, M Gates, A Iatrow, S Lang, S Bratton, Department of Pediatrics, University of Michigan, Ann Arbor, Michigan.

Neonatal intensive care unit (NICU) practices may influence the delivery of ophthalmology care for infants eligible for back transfer to a referring community hospital. We conducted this study to assess barriers to screening for Retinopathy of Prematurity (ROP) after discharge or transfer from a NICU. Study Design: Retrospective study of 78 infants who needed ophthalmology examinations at the time of their discharge o transfer from the NICU. These infants either needed screening for ROP or had retinal examinations in the NICU and needed further follow-up. Results: $64 \%$ of infants received appropriate eye care. Infants who did not receive the follow-up care had greater mean gestational age (mean SD; $30.7 \pm 2.3$ vs. $29.4 \pm 2.6$ weeks, $p=0.02$ ) and birth weights (mean SD; $1581 \pm 366$ vs. $1329 \pm 504 \mathrm{gm}, \mathrm{p}=0.007$ ) compared to infants who received the recommended care. There were no statistical differences between the two groups when comparing their race, maternal age, maternal marital status, insurance coverage, or site of birth. Forty-three $(55.1 \%)$ infants were transferred back, $26(33.3 \%)$ infants were discharged home from the NICU, while nine (11.6\%) infants were discharged home from the regional center after their transfer to the general pediatrics service. Infants discharged from the NICU were significantly more likely to receive recommended eye care compared to infants transporte back to the community hospital or transferred to the pediatric service at the regional center (Relative Risk Ratio $1.5,95 \%$ confidence interval $1.1-2.1, \mathrm{p}=0.01)$. Infants transferred or discharged from the NICU not screened for ROP $(\mathrm{n}=49)$ had lower completion rates compared to infants who had their first retinal examination in the NICU and needed follow-up $(n=29)(89 \%$ vs. $49 \%, p<0.0001)$. Infants whose ophthalmology follow up was recommended in the discharge summary and had their appointments arranged prior to discharging them from the community hospital or the regional center were ten time more likely to get the appropriate eye care (Relative Risk Ratio 10.7, 95\% Confidence Interval 2.8-40.3, p <0.0001) compared to infants who did not have arrangements and recommendations. Parents were compliant $(\mathrm{n}=56,89 \%)$ with pre-arranged appointments. Conclusions: Infants transferred back or discharged from the NICU before having a retinal examination represent a high-risk group for not receiving eye screening. Scheduling appointments for these infants when they leave the NICU may improve their ROP screening and care.

\section{7}

TRANSIENT HYPERAMMONEMIA IN PRETERM INFANTS WITH HYPOXIA.

G Brar, R Thomas, EV Bawle, V Delaney-Black, Division of Neonatology, Wayne State University, Detroit, MI.

Purpose of study- Transient hyperammonemia of the newborn can lead to an overwhelming and potentially fatal illness. The etiology of this condition is obscure although hypoxia, immature urea cycle and inadequate hepatic blood flow have all been implicated. We hypothesized that preterm infants with hypoxia would have elevated ammonia levels after birth that normalize within 3-4 weeks. The study aim was to measure seria plasma ammonia levels in preterm infants with hyaline membrane disease (HMD) or perinatal asphyxia to elucidate the role of hypoxia in the pathogenesis of hyperammonemia. Methods- Infants 24-37 weeks gestation with severe HMD or perinatal asphyxia who had indwelling umbilical catheters and parental consent were enrolled. Hyperalimentation with $10 \%$ trophamine was initiated at $24-48$ hours of life. Blood samples were drawn at 24 hours, 48 hours and weekly until ammonia levels normalized. Plasma ammonia was analyzed using Vitros AMON Slide Analyzer. A level $\geq 50 \mathrm{umol} / \mathrm{l}$ was considered elevated. Pregnancy and neonatal data were collected. Repeated measures ANOVA was employed to examine mean differences in serial plasma levels in each group. All analyses were conducted using SPSS Version 11.5. Results- The study sample consisted of 20 neonates in the HMD and 6 in the asphyxia group. Infants with asphyxia had significantly lower apgar scores, cord $\mathrm{pH}$ and higher ALT levels. In the HMD group, mean plasma ammonia level decreased from $100.68 \pm 28.35$ umol/l at 24 hours to $88.42 \pm 23.61$ umol/l at 48 hours $(\mathrm{p}=0.003)$ and $56.84 \pm 18.42 \mathrm{umol} / /$ at week $2(\mathrm{p}<0.001)$. In the perinatal asphyxia group, mean ammonia levels decreased from $97.67 \pm 10.78 \mathrm{umol} / \mathrm{l}$ at 24 hours to $88.67 \pm 9.02 \mathrm{umol} / \mathrm{l}$ at 48 hours $(\mathrm{p}=0.003)$ and $56.83 \pm 7.12 \mathrm{umol} / \mathrm{l}$ at week $2(\mathrm{p}<0.001)$. No significant between group differences were seen in mean plasma ammonia. Ammonia levels correlated significantly with $\mathrm{pH}$ (neg), pO2(neg) and FiO2 (pos). Multiple regression analyses revealed that both $\mathrm{pO} 2$ and $\mathrm{pH}$ accounted for $\sim 73 \%$ variance of plasma ammonia levels $(\mathrm{p}<0.001)$ and $\mathrm{pO} 2$ alone accounted for $\sim 52 \%$ variance. ConclusionsAmmonia levels decreased significantly from 24 hours to week 2 in both groups. No significant correlation of plasma ammonia levels was seen with birth weight, gestational age, apgar scores, glucose, calcium, bilirubin, ALT levels and total caloric and protein intake. Hyperammonemia was not associated with neurologic dysfunction. PO2 and $\mathrm{pH}$ significantly predicted ammonia levels. Thus, hypoxic state associated with HMD and perinatal asphyxia is associated with transient hyperammonemia that lasts for $2-3$ weeks.

\section{8}

NUTRITION PRACTICE GUIDELINES AND NEONATAL OUTCOMES IN ELBW INFANTS.

R. Donovan, B. Puppala, D. Angst, B. Coyle, Advocate Lutheran General Children's Hospital, Park Ridge, IL.

Early nutritional intervention, both parenteral and enteral, is becoming a standard of care for the extremely low birth weight infant (ELBW) in many neonatal intensive care units (NICU) across the country. However, there are no published or widely accepted guidelines regarding nutrition support strategies for this population. Most NICU's have developed their own guidelines and nutritional practices vary widely. In an effort to standardize our practice, we implemented nutrition support guidelines for ELBW infants, initiating both total parenteral nutrition (TPN) and minimal enteral feedings (MEFs) within the first 24 hours of life whenever possible. Objectives: 1) Evaluate the adherence to the nutritional guidelines and 2) Compare pre- and postguideline outcomes. Materials and Methods: The study was conducted at a Level III NICU from January 2002 until February 2003. Charts of 70 infants born $\leq 1250 \mathrm{gms}$ were reviewed as part of a quality assurance project to monitor adherence to the newly established guidelines. Another 23 charts of ELBW infants who were admitted and cared for in the NICU prior to the initiation of the nutritional guidelines were reviewed as a control group. Student $t$-tests were used to compare selected clinical outcomes between infants started on early nutrition support ( $\leq 24$ hours of life) versus those who were started later. Results: $61.4 \%$ and $52.9 \%$ of eligible infants were started on TPN and MEF's, respectively within 24 hours of life. The average time to start TPN was 21.9 In the post-guideline group, MEF's were initiated at mean 27.3 hours of age versus 80.3 hours in the pre-guideline group $(\mathrm{p}<0.01)$. Those who were started on early TPN and MEF's reached full enteral feedings significantly sooner (mean:12.8 and 13.3 days vs. 45.8 and 45.8 days, respectively; $p<0.01$ ). Early nutrition significantly sooner (mean: 12.8 and 13.3 days vs. 45.8 and 45.8 days, respectively; $\mathrm{p}<0.01$ ). Early nutrition
support also resulted in earlier regain of birth weight (mean day 12.7 vs. 16.0 for early vs. late TPN; $\mathrm{p}<0.01$ and mean day 13.8 vs. $16.0 ; \mathrm{p}<0.04$ for early vs. late MEF's). While not statistically significant, infants who received earlier nutrition support showed trends toward greater overall weight gains in weeks 2-3 and 3-4o life and a lower incidence of elevated serum blood glucose for infants who received earlier nutrition support. Conclusions: The implementation of early Nutrition support guidelines influenced the timeliness of initiating nutrition support in our unit. Early initiation of TPN and MEF's in ELBW infants produces a more rapid regain of initial weight loss, improves weight gain and, enhances earlier achievement of full enteral feedings. hours after the adoption of the guidelines as opposed to 64.4 hours prior to guideline implementation $(\mathrm{p}<0.01)$ 